\title{
STUDIES ON THE GALACTIC DENSITY WAVE
}

\author{
JA MES W.-K. MA RK \\ Massachusetts Institute of Technology, Cambridge, Mass., U.S.A.
}

\begin{abstract}
For spiral galaxies, investigations have been made as to the behaviour of density waves at the critical regions of the Lindblad and co-rotation resonances and in particular as to their role in the maintenance and generation of these waves. We shall also discuss the conditions under which an inner resonance ring may be formed. Furthermore, we find that the co-rotation region acts as a wave-amplifier.
\end{abstract}

\section{Inner Resonance and Resonance Rings}

Consider a galaxy with $\Omega$ and $\kappa$ denoting its circular and epicyclic frequencies as a function of radial distance from the galactic centre. The inner Lindblad resonance occurs when $\left(\Omega-\Omega_{\mathrm{p}}\right)=\kappa / m$, where $\Omega_{\mathrm{p}}$ is the constant pattern frequency of a density wave with number of arms $m$. This resonance is known to be very efficient in absorbing the inward-propagating galactic density waves (Toomre, 1969; Mark, 1971).

For the Schmidt model of our Galaxy, using the pattern speed of $\Omega_{\mathrm{p}}=13.5 \mathrm{~km} \mathrm{~s}^{-1}$ $\mathrm{kpc}^{-1}$ (Lin et al., 1969), we found (Mark, 1971) that the wave has a peak at about $5 \mathrm{kpc}$. This correlates well with the peak in the observed ratio of neutral to ionized hydrogen. Lin and Feldman (1970) had first suggested such a correlation as a consequence of the density wave theory but the exact position of the wave peak was not known to them. We must emphasize that our theory predicts that the position of this resonance 'ring' of ionized hydrogen is usually one to two kiloparsecs outwards of $r_{\mathrm{L}}$, the radius at which the resonance condition is satisfied, since the stars which are in resonant interaction with the wave can have radial excursions due to epicyclic motions even though their average radial distance may be nearly equal to $r_{\mathrm{L}}$. Figure 1 illustrates this situation.

An interesting property of the wave at inner resonance is its behaviour when the dispersion speed of the stars is varied. For low dispersion speeds, the wave has very short wavelengths in the region of inner resonance and should give the impression of a 'ring' when the thickness of the gaseous arms (in ionized hydrogen) is taken into account. Also the wave amplitude is larger at the ring than in the spiral structure further out. Thus we expect the ratio of ionized to neutral hydrogen to have a peak corresponding to the wave peak. But if the dispersion speeds are large, the spiral structure is more open and the wave is absorbed without ever winding up. We expect here no 'ring' structure in the density of ionized hydrogen and only a smooth decay of the spiral structure inwards through this region. In intermediate cases, the ring may be only partially closed. These expectations should of course be checked by a calculation of the relation of wave amplitude to ionized hydrogen density.

Short-wavelength leading waves convect outwards, with decreasing amplitude in this direction. No sharp peak in wave amplitude can form in the resonance region. Such waves can be excited by mechanisms located near the central region of galaxies. 


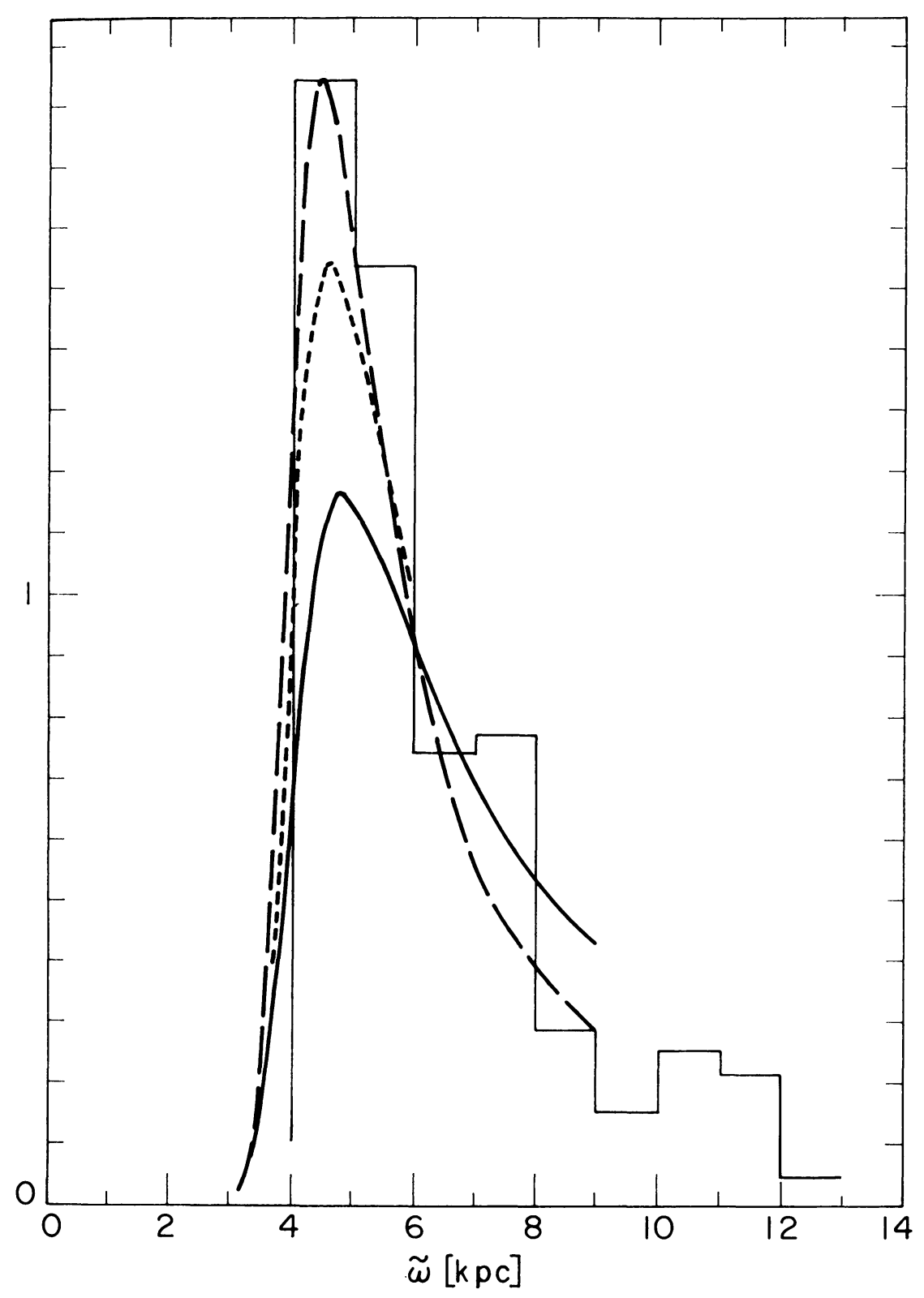

Fig. 1. The plot of observed $\mathrm{HII} / \mathrm{H}$ I density ratio versus radial distance is compared with three theoretical curves of wave surface mass density (multiplied by a geometrical factor). Mezger's (1969) number density of $\mathrm{H}_{\mathrm{I}}$ regions is used for the ionized hydrogen, while the $\mathrm{H}$ I density is that obtained by van Woerden (1965). The theoretical curves are obtained using the 1965 Schmidt model and $\Omega_{1} \ldots 13.5 \mathrm{~km} \mathrm{~s}^{-1} \mathrm{kpc}^{1}, r_{1}, 3.2 \mathrm{kpc}$. The solid, dotted and dashed curves represent a sequence of decreasing velocity dispersion for the stars. 


\section{Inner Resonance and the Mechanism of Absorption}

We have also found that the details of this absorption of wave angular momentum (action) can be described by a continuity equation which includes source terms (i.e. a wave-action principle). In particular, the wave properties in this region are completely described by the density, flux and source of wave action respectively. For example, the action density is negative for the short-wavelength trailing waves and these waves convect inwards towards the inner resonance. The source density is always positive and thus contributes only to the damping of the wave. As this wave convects inwards, the action density first increases in magnitude because the wave

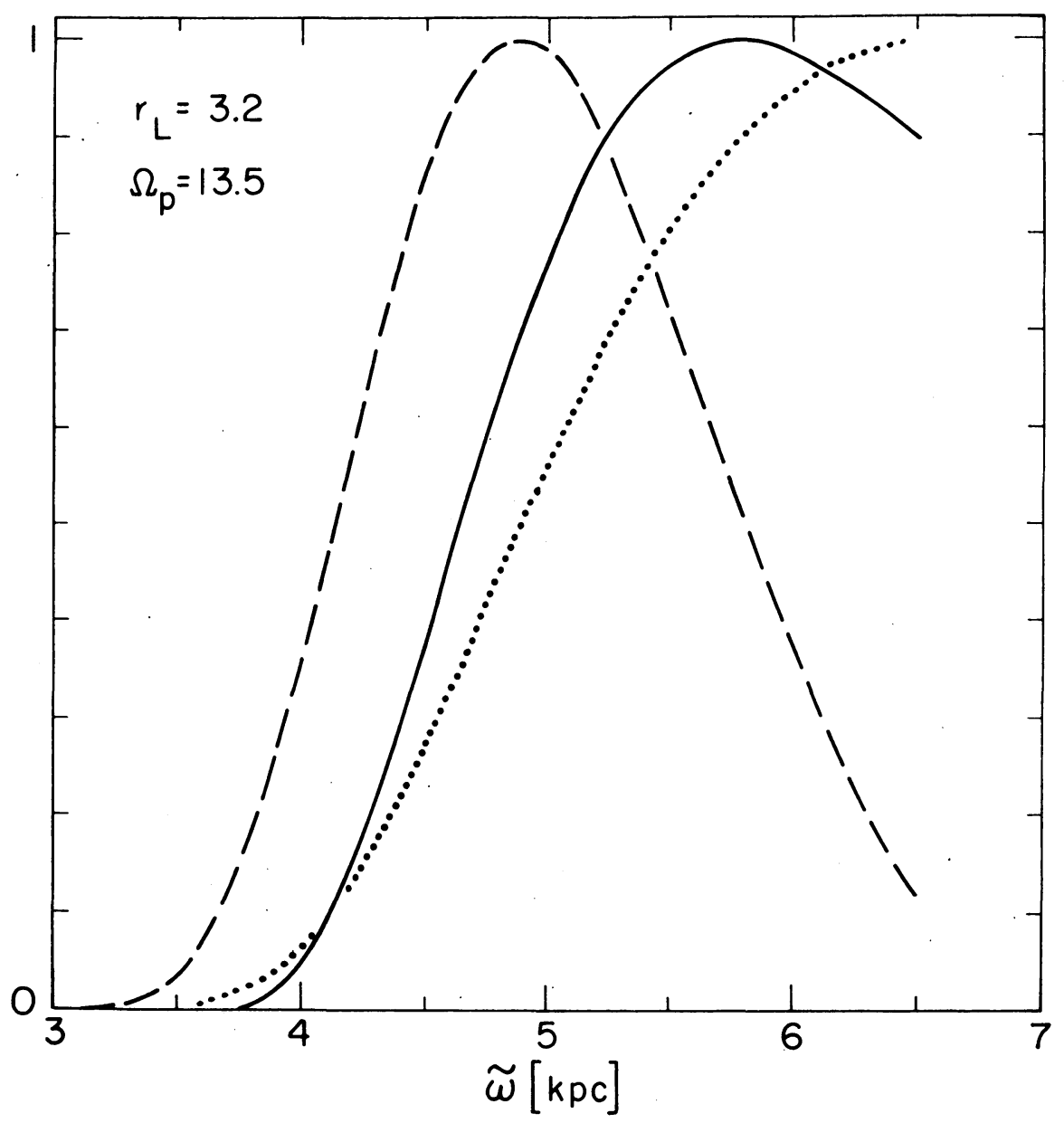

Fig. 2. Plotted against radial distance from the galactic centre are the magnitude of the wave angular momentum density (solid curve), the wave angular momentum flux (dotted curve) and the source density (dashed curve) of angular momentum that damps the wave at inner Lindblad resonance. The model used and pattern speed are the same as in Figure 1, while the vertical scale is in arbitrary units. 
flux decreases. But eventually, at about $2-3 \mathrm{kpc}$ outwards of the radius $r_{\mathrm{L}}$, the magnitude of the action density decreases sharply inwards because of the source. By the time the radius $r_{\mathrm{L}}$ is reached, the wave is completely negligible in amplitude. The behaviour of the relevant physical quantities is illustrated in Figure 2 for this case of short-wavelength trailing waves.

Within the approximations used, the integrated source strength agrees with that supplied by Lynden-Bell and Kalnajs (1972). Thus we have confirmed by detailed calculation that their mechanism for absorption is correct.

\section{Preliminary Results on the Possibility of Forming a Bar-Like Density Concentration at the Inner Resonance Region*}

Lin (1969) has suggested a mechanism for maintaining the wave which requires the driven response of the inner resonance to possess a bar-like component. Contopoulos (1970) had found a four-fold symmetry in the resonant periodic and tube orbits. He used a wave of nearly constant amplitude. We have recalculated the stellar orbits in this 3-5 kpc region of the galaxy, assuming steady driving by a density wave which takes into account the rapid decay of the wave amplitude inside $5 \mathrm{kpc}$.

The resonant periodic orbits are individually still roughly elliptical in shape, in agreement with Contopoulos' (1970) calculations for constant wave amplitude. We differ from him in that all these resonant orbits have major axes pointing in the same general direction with deviations up to some $30^{\circ}$. This suggests that a bar-like density concentration has formed. In addition to this, many of the non-periodic orbits tend to suggest the presence also of a density with fourfold symmetry.

However, although the above results are suggestive, it is not easy to determine from them whether the density concentrations are large enough to provide the necessary feedback. A continuum-type calculation is now being pursued which may hopefully give us some more definite numerical estimates on the actual density concentrations.

\section{The Co-Rotation Region and an Amplifier of Density Waves}

Lynden-Bell and Kalnajs (1972) suggested that the co-rotation resonance may be a source of negative wave action which excites the inward-travelling trailing waves. We studied this resonance for the case where the galactic disk is neutrally stable to Jeans' instability. In order to obtain a consistent solution near this resonance, it turned out to be necessary to consider a process of wave reflection and transmission rather than the simple interaction of one wave with the resonant stars as envisaged by Lynden-Bell and Kalnajs.

At the co-rotation resonance an outward-travelling trailing wave is reflected and transmitted into other trailing waves. The wave generation effect suggested by LyndenBell and Kalnajs resulted only in a slight amplification of the reflected wave relative

* The work in this section is performed in collaboration with Mr Robert Berman. 
to the incident one. An inward-travelling trailing wave is similarly reflected and transmitted at the co-rotation resonance. We used the Schmidt model of our Galaxy.

However, another mechanism is operating at the co-rotation region which acts as an amplifier of wave energy (or angular momentum). This new mechanism depends on the presence of negative energy waves inside co-rotation and positive energy waves outside co-rotation. In the above-mentioned reflection and transmission process, these waves of different intrinsic energy content are coupled across the co-rotation

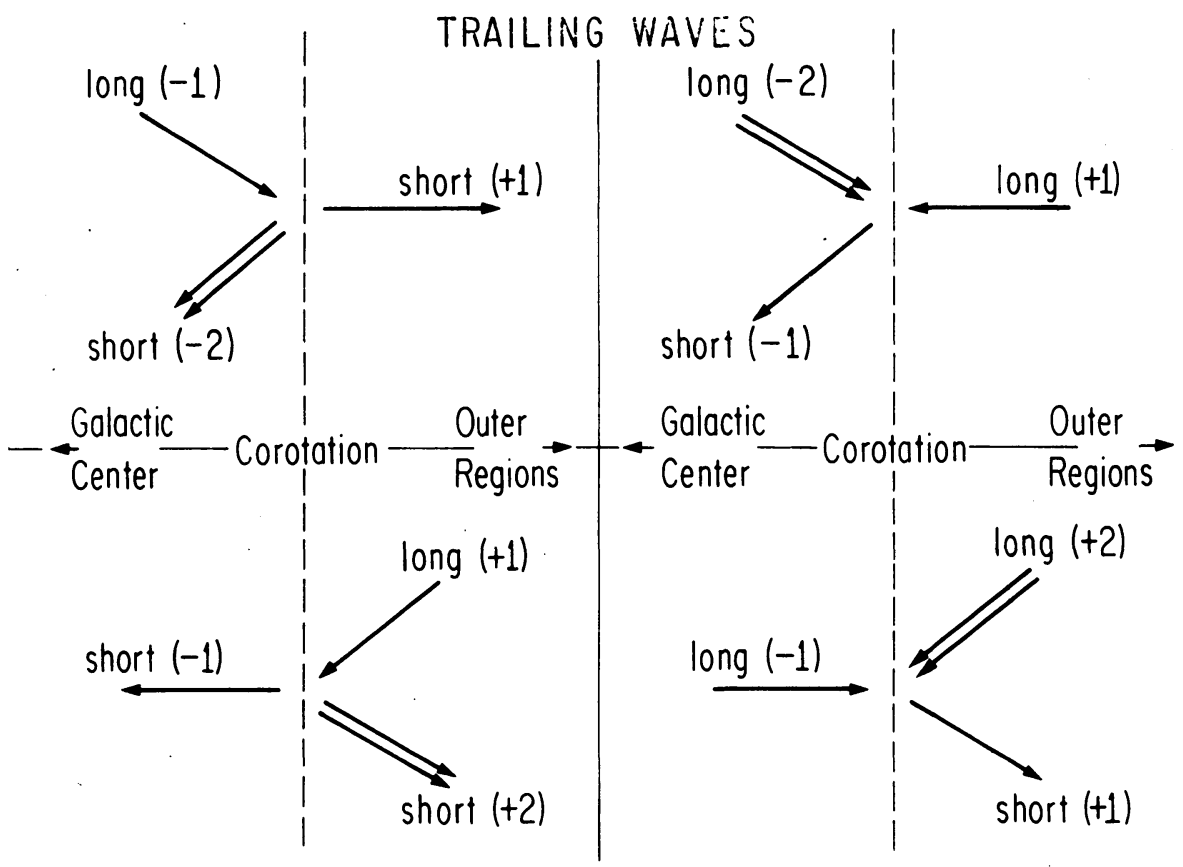

Fig. 3. Schematic diagram of two wave amplifiers (left frames) and two 'de-amplifiers' (right frames). The co-rotation circle (dashed line) separates central and outer regions of the galaxy. Arrows indicate the direction and strength of the waves. The strength (energy content) is also given in arbitrary units by the signed numbers that follow the words 'long' or 'short' which identify the waves as members of the long or short wavelength branches.

region. Omitting the small contribution of the resonant stars, the reflected wave has twice the energy of the incident one, while energy conservation requires that the transmitted wave carries away an amount of energy equal in magnitude but opposite in sign to that carried by the incident wave. Figure 3 describes in a schematic fashion the four situations that occur for this particular case where the galactic disk has only a stellar component and where this is neutrally stable to axisymmetric Jeans' instability.

This wave amplification process provides one detailed mechanism whereby galaxies convert their abundant source of kinetic energy (and angular momentum) of circular rotation into the energy (and angular momentum) of the spiral wave patterns. The conversion occurs at the co-rotation region where the wave pattern frequency $\Omega_{\mathrm{p}}$ is 
nearly equal to the circular frequency $\Omega$ of the local stars. Such a process depends crucially on the fact that the major density waves (i.e., those inside of co-rotation) represent disturbances whose excitation requires the removal of wave energy (in the inertial frame). Amplification occurs due to the above-mentioned coupling with other waves which have the opposite behaviour in that their excitation requires an input of wave energy. Thus we may expect higher amplification to be possible if other 'dissipative' mechanisms occur which satisfy the criterion that they can remove rotational kinetic energy from the galactic disk. A slowly rotating galactic halo can preferentially remove rotational energy from the rapidly rotating disk and further wave amplification is found. Moreover, since the presence of halo stars and of the gas in the disk both increase the effectiveness of wave coupling in the above-described wave amplifier, therefore wave amplification is further enhanced. After much analysis, it is found that the behaviour of the waves at co-rotation can be described by the turning point equation

$$
\frac{\mathrm{d}^{2} w(\zeta)}{\mathrm{d} \zeta^{2}}+\left[\zeta^{2}+m_{\mathrm{D}}+m_{\mathrm{H}}+\varrho(\zeta)\right] w(\zeta)=0
$$

Here, $\zeta$ measures (scaled) distance away from co-rotation; $w(\zeta)$ is the reduced wave function; $m_{\mathrm{D}}$ and $m_{\mathrm{H}}$ are respectively the disk and halo amplification coefficients; and $\varrho(\zeta)$ gives the corrections due to the resonant stars at co-rotation. Figure 1 corresponds to the mathematically simpler case where $m_{\mathrm{D}}=m_{\mathrm{H}}=0$ and also $\varrho(\zeta)$ is omitted. But under the actual circumstances applicable to galaxies, the values of these coefficients are such that wave amplifications of at least an order of magnitude should be possible.

\section{References}

Contopoulos, G.: 1970, Astrophys. J. 160, 113.

Lin, C. C.: 1969, in W. Becker and G. Contopoulos (eds), 'The Spiral Structure of Our Galaxy', IAU Symp. 38, 377.

Lin, C. C. and Feldman, S. : 1970, Ref. C. C. Lin, Highlights of Astron. 2, 88, 1971.

Lin, C. C., Yuan, C., and Shu, F. H.: 1969, Astrophys. J. 155, 721.

Lynden-Bell, D. and Kalnajs, A.: 1972, Monthly Notices Roy. Astron. Soc. 157, 1.

Mark, J. W-K.: 1971, Proc. Nat. Acad. Sci. 68, 2095.

Mezger, P. G.: 1969, in W. Becker and G. Contopoulos (eds), 'The Spiral Structure of Our Galaxy', IAU Symp. 38, 107.

Toomre, A.: 1969, Astrophys. J. 158, 899.

van Woerden, H.: 1965, Ref. J. H. Oort, Trans. IAU 12A, 789.

\section{DISCUSSION}

Contopoulos: The effect of a density wave upon particles originally moving in circular or epicylic orbits will be to change their orbits and, if strong enough, to 'trap' them in non-linear, resonant, quasi-periodic orbits. This 'trapping' process is probably the one normally responsible for absorption of the waves at the inner Lindblad resonance and for the excitation of the waves at the co-rotation distance. 
Mark: I agree that trapping is the mechanism causing absorption of the waves at the inner resonance, but $\mathrm{I}$ have come to the conclusion that the trapping process at the co-rotation resonance is not effective in exciting the wave. My solutions seem to be different from that of Dr Contopoulos. For trailing waves, one solution is that of a long wave propagating outwards, which becomes reflected and transmitted at the co-rotation region into two short trailing waves. 